\title{
Dysphagia Management in Head and Neck Cancers: a Manual and Atlas
}

https://doi.org/10.32635/2176-9745.RBC.2020v66n4.1235

\author{
Manejo da Disfagia no Câncer de Cabeça e Pescoço: Manual e Atlas \\ Manejo de la Disfagia en Cánceres de Cabeza y Cuello: Manual y Atlas
}

Thankappan K, Iyer S, Menon JR, editors. Singapura: Springer; 2018. 404p.
ISBN 978-981-10-8281-8 (versão impressa)
ISBN 978-981-10-8282-5 (eBook)

José Alfonso Vergara Herazo'; Guilherme Maia Zica²

O câncer de cabeça e pescoço (CCP) representa as neoplasias malignas das vias aerodigestivas superiores e afeta principalmente indivíduos do sexo masculino, acima de 40 anos de idade e com consumo associado de tabaco e álcool. No Brasil, o CCP é o $5^{\circ}$ tipo de câncer mais frequente em homens e o $13^{\circ}$ mais frequente em mulheres ${ }^{1}$.

A deglutição é o processo de transporte do alimento da boca até o estômago e sua disfunção denomina-se disfagia ${ }^{2}$. É consenso na literatura que o paciente com CCP apresenta alto risco para o desenvolvimento de disfagia em razáo do comprometimento de regiôes anatômicas importantes para a dinâmica orofaríngea ${ }^{3}$. A equipe multiprofissional pode auxiliar e dar suporte para esse paciente em todos os estágios do tratamento ${ }^{4}$.

Recentemente, foi lançado o livro Dysphagia Management in Head and Neck Cancers: a Manual and Atlas (em português: Manejo da Disfagia nos Cânceres de Cabeça e Pescoço: Manual e Atlas) publicado pela editora Springer Singapura, em 2018, com 404 páginas, e organizado pelos cirurgiōes de cabeça e pescoço Krishnakumar Thankappan e Subramania Iyer e pelo otorrinolaringologista Jayakumar R. Menon. Todos fazem parte do Departamento de Oncologia e Cirurgia de Cabeça e Pescoço do Instituto de Ciências Médicas Amrita, Kochi, na Índia; além disso, contam com uma longa experiência no tratamento e acompanhamento de pacientes com disfagia decorrentes de CCP. Este livro apresenta um compilado de contribuiçóes e textos de mais de 30 especialistas na área de oncologia de cabeça e pescoço, possibilitando a visão interdisciplinar do tema.

O livro está organizado em seis partes, com diferentes capítulos, que trazem uma compilação das referências mais atuais. Cada parte apresenta-se com uma descrição, apresentação e divisão das informações que fazem parte de seu conteúdo com um recurso funcional, que não precisa (exclusivamente) ser lido do princípio ao fim, pois funciona como uma consulta rápida para o manejo das dificuldades encontradas durante a prática clínica. O livro é apresentado em forma de manual e atlas, disponibilizando recursos multimídia como imagens, vídeos e fotos, assim como estudos e discussôes de casos.

A parte I possui dois capítulos sobre a anatomia da cabeça e pescoço; disponibilizando tabelas com informaçóes detalhadas sobre os músculos, inervações e funções; da mesma forma, mapas e ilustrações full color são incluídas. Os conceitos de deglutição normal e alterada são trazidos com uma explicaçáo completa. O material desenvolvido pelos autores descreve a deglutição em toda sua complexidade por meio de esquemas biomecânicos e mapas mentais da fisiologia, anatomia e controle neural dessa função. É evidente a preocupação com a compreensão da biomecânica da deglutição, em busca de fundamentar o leitor para traçar um raciocínio adequado nas alterações provenientes do tratamento oncológico. Tudo isso com recursos visuais de fácil localização, visando a favorecer os estudos e a leitura rápida para os profissionais de saúde.

A parte II conta com seis capítulos que descrevem os métodos de avaliação da deglutição, começando pela avaliação pré-tratamento e discutindo os achados que podem ser preditivos para o prognóstico da disfagia após o tratamento. São descritos os métodos clínicos (avaliação clínica da deglutição) e os instrumentais (videofluoroscopia, nasofibrolaringoscopia da deglutição, esofagoscopia transnasal e manometria); os autores discutem as formas de

'Fonoaudiólogo. Mestrando em Ciências da Cirurgia na Faculdade de Ciências Médicas da Universidade Estadual de Campinas (Unicamp). Campinas (SP), Brasil. Orcid iD: https://orcid.org/0000-0001-9922-9347

${ }^{2}$ Fonoaudiólogo. Mestrando em Distúrbios da Comunicação Humana da Universidade Federal de São Paulo (Unifesp). São Paulo (SP), Brasil. Orcid iD: https://orcid. org/0000-0003-4546-4063

Endereço para correspondência: Guilherme Maia Zica. Universidade Federal de São Paulo (Unifesp). Rua Sena Madureira, 1500 - Vila Clementino. São Paulo (SP), Brasil. CEP 04021-001. E-mail: guilhermamaiafono@gmail.com 
realização desses instrumentos e destacam algumas recomendaçôes. A informação completa possibilita que o leitor adapte seus métodos de avaliação de acordo com os recursos presentes em seu serviço e capacita o profissional para a coleta de dados e descrição do perfil funcional.

$\mathrm{Na}$ parte III do livro, ao longo de 11 capítulos, discutem-se os princípios gerais do manejo da dieta por via oral, assim como as possibilidades de reabilitação (exercícios e terapias compensatórias). Os capítulos são autoexplicativos e possuem ilustraçôes, fotos e vídeos dos exercícios e manobras de reabilitação, assim como tabelas que resumem essas técnicas, o que facilita a compreensão da informação. Algumas consideraçóes nutricionais são abordadas, discutem o uso vias alternativas de alimentação (sondas de alimentação, gastrostomias, jejunostomias), oferecendo princípios norteadores que facilitam identificar os potenciais casos que podem se beneficiar dessas abordagens. Os autores dão alta relevância aos fatores sociais e psicológicos, e destacam a importância de uma abordagem abrangente que vise ao bom impacto na qualidade de vida do paciente. Outros temas mais atuais e controversos também são abordados, como a eletroestimulação, o que capacita o leitor para o desenvolvimento de um raciocínio clínico e crítico por meio de embasamento científico.

A parte $I V$ do livro trata sobre a disfagia após cirurgias de cavidade oral, orofaringe, laringe e hipofaringe; mostrando vídeos e imagens dos resultados nas avaliações instrumentais da deglutição de acordo com o tipo de cirurgia e abordando, dessa mesma forma, os diferentes tipos de condutas terapêuticas (exercícios, manobras e compensaçôes). Além disso, os autores estudam o uso de cirurgias reconstrutivas em CCP considerando aspectos estéticos, os quais são de vital importância para o menor impacto na qualidade de vida.

Após o tratamento exclusivo com radioterapia/quimioterapia (ausência de cirurgia), a disfagia também pode estar presente; esse tema é abordado na parte $V$, na qual são apresentadas as estratégias para prevenir e reabilitar a disfagia associada com a radioterapia. Diferentes exercícios profiláticos baseados em estudos científicos são abordados e casos clínicos, apresentados para a aplicação prática do conhecimento. O manejo de outros sintomas como a mucosite e a dor são tratados amplamente. O material torna-se de grande valia, dentro de um contexto ainda pouco estudado no mundo e aplicado à prática.

O livro finaliza com a parte VI apresentando um resumo do conteúdo e oferecendo algumas ideias norteadoras sobre as pesquisas necessárias no tópico aqui abordado.

O livro Dysphagia Management in Head and Neck Cancers: a Manual and Atlas ${ }^{5}$, pela riqueza do seu conteúdo, está destinado a todos os profissionais envolvidos no manejo da disfagia no paciente com CCP. O material é atual e oferece descriçôes práticas que podem facilitar a prática clínica com uma linguagem clara, além de recursos multimídia que favorecem o aprendizado. É um material desenvolvido na Índia, país que apresenta contexto socioeconômico e de saúde próximo ao do Brasil, o que evidentemente favorece um conteúdo aplicável à nossa realidade.

Os fonoaudiólogos e profissionais da saúde que prestam atendimento a essa população precisam ter um entendimento abrangente das diferentes formas de disfagia no CCP, fatores associados ao risco elevado de desenvolver complicações e objetivos do paciente. Quando a tomada de decisão ponderada é aplicada no tratamento da disfagia associada ao CCP, a função e a qualidade de vida são otimizadas ${ }^{2,4}$.

\section{REFERÊNCIAS}

1. Instituto Nacional de Câncer José Alencar Gomes da Silva. Estimativa 2020: incidência de câncer no Brasil [Internet]. Rio de Janeiro: INCA; 2019 [acesso 2020 abr 30]. Disponível em: https://www.inca.gov.br/publicacoes/livros/estimativa2020-incidencia-de-cancer-no-brasil

2. Starmer HM. Dysphagia in head and neck cancer: prevention and treatment. Curr Opin Otolaryngol Head Neck Surg. 2014 Jun;22(3):195-200. doi: https://doi.org/10.1097/MOO.0000000000000044

3. Chen SC. Oral dysfunction in patients with head and neck cancer: a systematic review. J Nurs Res. 2019;27(6):e58. doi: https://doi.org/10.1097/jnr.0000000000000363

4. Denaro N, Merlano MC, Russi EG. Dysphagia in head and neck cancer patients: pretreatment evaluation, predictive factors, and assessment during radio-chemotherapy, recommendations. Clin Exp Otorhinolaryngol. 2013 Sep;6(3):11726. doi: https://doi.org/10.3342/ceo.2013.6.3.117

5. Thankappan K, Iyer S, Menon JR, editors. Dysphagia management in head and neck cancers: a manual and atlas. Singapura: Springer; 2018. doi: https://doi.org/10.1007/978-981-10-8282-5 